\title{
Acceptance of fluorescence detectors and its implication in energy spectrum inference at the highest energies
}

\author{
Vitor de Souza * Gustavo Medina-Tanco tit and Jeferson A. Ortidt \\ Instituto de Astronomia, Geofísica e Ciências Atmosféricas, Universidade de São Paulo
}

(Dated: October 21, 2018)

\begin{abstract}
Along the years HiRes and AGASA experiments have explored the fluorescence and the ground array experimental techniques to measure extensive air showers, being both essential to investigate the ultra-high energy cosmic rays. However, such Collaborations have published contradictory energy spectra for energies above the GZK cut-off. In this article, we investigate the acceptance of fluorescence telescopes to different primary particles at the highest energies. Using CORSIKA and CONEX shower simulations without and with the new pre-showering scheme, which allows photons to interact in the Earth magnetic field, we estimate the aperture of the HiRes-I telescope for gammas, iron nuclei and protons primaries as a function of the number of simulated events and primary energy. We also investigate the possibility that systematic differences in shower development for hadrons and gammas could mask or distort vital features of the cosmic ray energy spectrum at energies above the photo-pion production threshold. The impact of these effects on the true acceptance of a fluorescence detector is analyzed in the context of top-down production models.
\end{abstract}

PACS numbers: $96.40 . P q$

\footnotetext{
*Electronic address: vitor@astro.iag.usp.br

${ }^{\dagger}$ Electronic address: gmtanco@gmail.com

${ }_{\ddagger}^{\ddagger}$ Electronic address: jortiz@astro.iag.usp.br
} 


\section{INTRODUCTION}

Since the very first observations at the beginning of the $20^{\text {th }}$ century, ultra-high energy cosmic rays have been a priority in astroparticle physics and an open question. The flux of particles is one of the most intriguing question to be answered. Measurements of the energy spectrum of particles allows us to make assumptions about a great number of astrophysical models, such as, acceleration mechanisms, magnetic field in the source and in the medium, propagation mechanisms and anisotropy.

At energies above $10^{19.5} \mathrm{eV}$, the flux of particles is expected to drop abruptly due to the interaction of the particles with the microwave background. Such effect, widely known as the GZK cut-off 1, 2], imposes sources of cosmic rays in the neighborhood of approximately 100 Mparsec. Inside this region, only a few classes of astrophysical objects have been argued to be able to accelerate particles at these high energies. Moreover, the energy threshold for pion photoproduction on the microwave background is $\sim 2 \times 10^{10} \mathrm{GeV}$, and at $3 \times 10^{11} \mathrm{eV}$ the energy-loss distance is about $20 \mathrm{Mpc}[3]$.

Nevertheless, several experiments have published detections of cosmic ray particles with energies above the GZK cut-off. Presently, the AGASA [4] and HiRes [5] Collaborations are the experiments with highest aperture at energies above $10^{19.5} \mathrm{eV}$. The HiRes Collaboration has firmly settled the GZK cut-off prediction along the years. On the other hand, the AGASA Collaboration has argued for the non existence of the drop in the flux.

Many theoretical works have tried to overcome such scientific disagreement and production mechanisms have been proposed in order to explain the excess of high energy particles seen by the AGASA Collaboration in relation to the expected GZK cut-off. An explanation of the highest energy cosmic rays is the top-down scenario which considers the decay of relic particles of the early universe, i.e., topological defects or super-heavy particles which may decay into jets of hadrons, mainly pions that subsequently decay into $\gamma$-rays, electrons and neutrinos. Only a few percent of the particles produced by these mechanisms are hadrons which might result in $\gamma$-ray and neutrinos as the principal component generated in these process.

HiRes and AGASA experiments have explored different experimental techniques, the fluorescence and the ground array, respectively, to measure extensive air showers. The ground array efficiency is based on a straightforward detection given by its operational 
area which is, in principle, not dependent on the primary particle type. On the other hand, fluorescence telescopes measure the longitudinal air shower development by detecting the fluorescence light emitted along the track of particles in the atmosphere. Particles with different mass induce showers with distinct longitudinal evolution in the atmosphere and for this reason the efficiency of fluorescence telescopes depends on the primary shower composition.

The HiRes Collaboration states in reference [6] that the HiRes-I telescope aperture investigation has been based on simulations of proton and iron nuclei initiated showers and no mention to gamma-ray showers has been done so far. In the present work we calculate the HiRes-I telescope aperture for gamma showers in the energy range from $10^{19} \mathrm{eV}$ to $10^{20.6} \mathrm{eV}$. The corresponding spectrum for the bottom-up and top-down scenarios are calculated and compared to the AGASA data. Furthermore, we have studied the determination

of the aperture as a function of the number of simulated showers and for different simulation codes.

This paper is structured as follows. In Section III we briefly discuss the longitudinal development of extensive air showers induced by different primary particles. In Section III the shower and detector simulation schemes are described. The reconstruction procedure and the analysis, as well, are characterized in Section [V] while the HiRes-I telescope aperture is depicted in Section V. An spectrum exposition is made in Section VI Section VII summarizes our results.

\section{LONGITUDINAL DEVELOPMENT OF SHOWER INITIATED BY DIFFER- ENT PRIMARY PARTICLES}

Fluorescence telescopes measure the longitudinal development of extensive air showers by detecting the fluorescence light emitted along the track of particles in the atmosphere. Showers initiated by particles with different mass are known to have different longitudinal development in the atmosphere.

Since the original proposal of the Gaisser-Hillas function [7], many studies [8, 9] have shown that the number of charged particles $(N)$, as a function of the atmospheric depth $(X)$, in hadronic-induced air showers is well described by a four parameter $\left(N_{\max }, X_{0}, X_{\max }\right.$ 
and $\lambda$ ) function according to equation:

$$
N(X)=N_{\max }\left(\frac{X-X_{0}}{X_{\max }-X_{0}}\right)^{\frac{\left(X_{\max }-X_{0}\right)}{\lambda}} \exp \frac{X_{\max }-X}{\lambda}
$$

In equation 1 only $N_{\max }$ and $X_{\max }$ have clear physical meaning in which $N_{\max }$ is defined as the maximum number of charged particles in the shower while $X_{\max }$ represents the depth at which the maximum number of charged particles occurred. We suggest the reference 10] which is an interesting summary about the physical meaning of each parameter.

The HiRes Collaboration [1] has shown that their data are completely independent from the chosen values of $X_{0}$ and $\lambda$. Correspondingly, for the HiRes-I analysis they have fixed the values of these parameters to $-60 \mathrm{~g} / \mathrm{cm}^{2}$ and $70 \mathrm{~g} / \mathrm{cm}^{2}$, respectively.

Both $N_{\max }$ and $X_{\max }$ have been widely used to reconstruct measured extensive air showers and to determine shower primary particle, since the energy and mass composition can be directly estimated as a function of these parameters. In addition to the elongation rate method [12], the $X_{\max }$ parameter has also been used to distinguish showers initiated by photons from those initiated by hadrons [13].

Not long ago, a new phenomenon has been shown to play a important rule in the development of gamma showers. Gammas with energies above $10^{19.0} \mathrm{eV}$ can interact with the Earth magnetic field and produced pairs of electron/positrons which can, by inverse Compton effect, emit photons. Such photons induce an electromagnetic cascade before the shower reaches the atmosphere, i.e., a pre-shower. The geomagnetic field effect shows significant importance in the development of gamma-initiated air showers. The overall effect of the pre-shower, concerning the longitudinal development, is to distort the gamma shower so that they show an atmospheric development more similar to proton showers. Results and application of the pre-shower program can be seen in references [13, 14, 15, 16].

Figs. 1 and 2 show the $X_{\max }$ distribution of 100 showers originated by different primary particles. The pre-shower effect is illustrated by the $X_{\max }$ distribution at $10^{19.5}$ and $10^{20.5}$ $\mathrm{eV}$. At $10^{19.5} \mathrm{eV}$ the simulation of gamma showers with and without the pre-shower are almost identical and only a small reduction of $X_{\max }$ is seen. At $10^{20.5} \mathrm{eV}$ the separation between the distributions of $X_{\max }$ simulated with and without the pre-shower effect is very clear.

Fig. 2 also illustrates how the pre-shower effect approximates the development of a gamma 
shower to that of a proton shower. However, even at high energies the $X_{\max }$ distribution of gamma showers are distinguishable from proton showers.

The detection of one shower by a fluorescence telescope depends on the entire development of the shower in the atmosphere. In particular, for the HiRes-I telescope, it is possible to calculate the minimum number of particles in a shower as a function of depth which would trigger the photomultiplier camera. Considering a background noise of 40 photoelectrons per $\mu s$ in each photomultiplier and applying a simple trigger condition of signal higher than four times the noise, it is possible to calculate for a given geometry the minimum number of particles in the shower which would trigger the photomultipliers.

Fig. 3 illustrates median and one sigma limits of the the longitudinal development of showers initiated by proton and gamma primaries (with pre-shower effect). In the same plot, we show the threshold for a shower landing $25 \mathrm{~km}$ away from the telescope with zenith

angle of 45 degrees. It can be seen that the telescope has a higher probability of triggering under a proton shower than under a gamma shower.

Besides the trigger conditions, the events used to construct the spectrum must survive the severe quality cuts imposed by the reconstruction of geometry and energy. The reconstruction quality of a shower depends on the size of the seen track and, in particular, the energy reconstruction error increases markedly if $X_{\max }$ is not in the field of view of the telescope.

Following the ideas underlined here, we investigate in the following sections the details involved in the probability of detection and reconstruction of showers initiated by different types of primary particles.

\section{SHOWER AND DETECTOR SIMULATION}

We used Monte Carlo simulations to evaluate the aperture of the HiRes-I telescope. The simulations preformed for this work followed the same general procedure adopted by the HiRes Collaboration as published in [6] and [11]. In these articles, the HiRes Collaboration has published the aperture of the experiment and all the important parameters used in the shower and detector simulation.

Following the HiRes Collaboration prescription, we have performed a very detailed simulation of the cosmic ray showers and of the HiRes-I telescope. Showers were simulated using the CORSIKA [17] program with the QGSJet [18] model for hadronic interactions. The 
thinning factor of the simulation was set to $10^{-6}$ and the longitudinal profile were sampled in steps of $5 \mathrm{~g} / \mathrm{cm}^{2}$. Energy thresholds were set to $0.1 \mathrm{MeV}$, for electrons and photons, 0.3 $\mathrm{GeV}$ for hadrons and $0.7 \mathrm{GeV}$ for muons. Protons and gamma ray showers were simulated in the energy interval from $10^{19}$ to $10^{20.5} \mathrm{eV}$ in steps of 0.1 dex. For each energy and primary particle we simulated 100 events.

For gamma ray showers, we have simulated two sets: a) with the pre-shower effect b) without the pre-shower effect. A recent release of CORSIKA (version 6.2) includes the pre-shower effect [19].

The probability of interaction of gammas with the geomagnetic field depends on the relative orientation between the local field and the momentum of the photons. Therefore we have used in our simulations the Earth magnetic field for the HiRes site $\left(112^{\circ} \mathrm{W}\right.$ and $40^{\circ}$ N) as given by reference [20].

Each CORSIKA shower is used several times to save computational time by drawing a zenith angle and a core position. For the gamma showers simulated with the pre-shower effect we only draw a new core position keeping constant the zenith angle. This is necessary because the development of the shower depends on the relative direction of the magnetic field with the primary particle.

For proton and iron nuclei showers, besides using CORSIKA, we have also simulated a second set of events with the recently released CONEX program [21, 22]. This program is based on a hybrid simulation scheme combining fast numeric solutions of cascade equations and Monte Carlo calculations. The main advantages of the hybrid programs is the execution time 23]. We have used CONEX in order to: a) test it in comparison to CORSIKA, regarding specifically the determination of the aperture of fluorescence telescopes, b) study the aperture for iron nuclei showers and c) evaluate the possible biases introduced by the recycling of the same shower several times, a common approach when using full simulation codes. Since, CONEX takes on average only 1.5 minutes to simulate one shower in our current system, we were able to run 5000 different showers for some energies and compare the result with 100 different CORSIKA showers used 50 times each. Results are presented in Section $\mathrm{VA}$.

From the longitudinal development of the charged particles in the atmosphere simulated by CORSIKA or CONEX, it is possible to calculate the number of fluorescence photons along the shower path using the fluorescence yield as measured by Kakimoto et al. [24]. 
Despite the fact that recent publications [25, 26, 27] have shown it is more direct and even more accurate to use the longitudinal energy deposit of particles we have chosen to use the longitudinal particles development in order to repeat exactly the HiRes Collaboration calculations made in references $[6,11]$

We therefore wrote a computer program to simulated the HiRes-I telescope which propagates the fluorescence photons to the detector by taking into account scattering and transmission effects (Rayleigh and Mie) according to [28]. Atmospheric conditions at the HiRes site have been carefully measured and the relevant parameters can be found at [6, 11].

Fluorescence photons are generated taking into account the average energy deposit as a function of shower age, atmospheric pressure dependence, and missing energy [9].

A complete wavelength dependent calculation was done. The fluorescence spectrum is taken from Bunner et al. [29] and the corresponding normalization applied according to Kakimoto et al. [24]. A detailed simulation of the detector was performed including effective collection area, mirror reflectivity, filters transmission and phototube quantum efficiency as given in references [30, 31].

Figs. 4 and 5 shows a comparison between our simulation program, the HiRes-I data and the HiRes Collaboration simulations. The HiRes data and simulation have been extracted from [6]. We have calculated the impact parameter of the showers detected by the telescopes. The number of simulated showers was normalized to the number of detected events.

The good agreement between our simulations and the HiRes data shows that we were able to reproduce the most important features of the detector. The $\chi^{2}$ between our simulation and the data is 1.7 and 1.5 for $10^{19} \mathrm{eV}$ and $10^{19.5} \mathrm{eV}$, respectively, while the $\chi^{2}$ between the HiRes Collaboration simulation and the data is 1.6 and 1.5 at the same energies.

\section{RECONSTRUCTION AND ANALYSIS}

The reconstruction of the shower also followed the procedures described in references 6, 11]. Basically, the inverse process described in Section first the shower longitudinal profile and subsequently its energy.

The number of photoelectrons measured in each pixel in the detector is mapped backwards onto (a) the number of photons in the telescope, (b) the number of photons in the axis of the shower and, finally, (c) the number of particles in the shower. The telescope effi- 
ciency, atmospheric absorption, fluorescence yield and missing energies have been considered according to Section III and references there in.

A Gaisser-Hillas [7] function was fitted to the data according to the specifications in reference [6]. The $X_{\max }$ parameter was allowed to vary in $35 \mathrm{~g} / \mathrm{cm}^{2}$ steps between 680 and $900 \mathrm{~g} / \mathrm{cm}^{2}$. The $X_{0}$ parameter of the Gaisser-Hillas was fixed to $-60 \mathrm{~g} / \mathrm{cm}^{2}$, according to reference [11]

Fig. [6] shows a longitudinal profile of a proton shower as seen by the HiRes-I telescope according to our simulation, including noise effects not present in the original CORSIKA shower, and the longitudinal profile reconstructed from the detected signal.

Quality cuts are always needed to ensure an accurate reconstruction. We have required events to satisfy the conditions listed below as taken from reference [6]:

- Average number of photoelectrons per phototube greater than 25;

- Angular speed less than $3.33^{\circ} \mu s$

- Track arc-length greater than $8.0^{\circ}$

- Depth of first observed point less than $1000 \mathrm{~g} / \mathrm{cm}^{2}$

- Angle of the shower in the plane containing the shower axis and the detector greater than $120^{\circ}$

Showers which did not obey those conditions were rejected and excluded from further analysis. The specific influence of each cut in the final acceptance of the telescope is the subject of a detailed study under way at present.

\section{HIRES-I TELESCOPE APERTURE}

The telescope aperture is essential for the determination of the spectrum. In order to calculate the HiRes-I telescope aperture we made a study on the fluctuation of the aperture as a function of the number of events simulated and the energy of the primary particle.

We varied the number of showers used to calculate the aperture from 100 to 5000 by using each CORSIKA shower from 1 to 50 times. We calculated the aperture 100 times for each total number of events. Analyzing the distribution of the aperture values we defined 
the median, the $68.2 \%$ and $95.4 \%$ confidence levels. The error is defined as the size of the confidence region divided by the median aperture.

Fig. 7 illustrates the error as a function of the total number of events simulated for showers initiated by protons with primary energy $10^{19.5} \mathrm{eV}$. It can be seen that the error stabilizes around 2500 simulated showers.

We repeated the same procedure by calculating the error as a function of energy. Fig. 8 shows the variation of the error with energy when we used 5000 proton showers in the simulation. After this analysis, in order to keep the error small at all energies, we decided to use 5000 showers for the calculation of the aperture.

\section{A. The dependence of the aperture determination with the shower simulation} model

In order to investigate the influence of the shower simulation model on the determination of the HiRes-I telescope we used proton showers simulated with CORSIKA as well as with CONEX.

Fig. 9] shows the aperture for the HiRes-I telescope for both simulation schemes. First, the figure illustrates once again that our simulation of the HiRes-I telescope produces the same results obtained by the HiRes-I Collaboration, and second, that CORSIKA and CONEX give the same results concerning aperture calculations. However, despite the success of this hybrid program (CONEX) in reproducing the same aperture as given by CORSIKA, we would like to stress that the aperture calculation is a very indirect procedure to compare simulation models. More details confrontations between full Monte Carlo and hybrid simulations have been done as can be seen in [23, 32].

Fig. 10] shows the aperture for the HiRes-I telescope calculated with CORSIKA and CONEX. For the energies of $10^{19}, 10^{19.5}, 10^{20}$ and $10^{20.5} \mathrm{eV}$ we have simulated a second set of 5000 different showers with the CONEX program. Fig. 10 shows the comparison between the two approaches: a) $(100 \times 50) 100$ different showers used 50 times each one by randomly

choosing a different geometry and b) (5000) 5000 different showers. The later approach was only possible with CONEX because it is much faster than CORSIKA. No significant difference between the two methods was seen. 


\section{B. The HiRes-I aperture for different primary particles}

We have also investigated the dependence of the aperture on the nature of the primary particles. According to the article published by the HiRes Collaboration [6] they have only investigated the aperture of the HiRes-I telescope for proton and iron nuclei showers and based on this aperture they have determined the spectrum.

Fig. 11 shows the aperture for showers initiated by proton, iron nuclei, gamma without the pre-shower effect and gamma with the pre shower effect. In all curves, we have used 100 different showers recycled 50 times each as explained in Section III.

It is noticeable that the HiRes-I telescope has, within fluctuations, the same aperture for hadronic showers: proton and iron nuclei. However, the aperture for gamma showers is well bellow the hadronic limit.

If the pre shower effect is not taken into account, gamma showers tends to develop much deeper in the atmosphere, as illustrated in Fig. [3, when compared to proton showers. The late development make the gamma showers simulated without the pre shower effect harder to detect and a great reduction in the telescope aperture is seen for all energies.

When the pre shower effect is taken into account, gamma shower with energy above $10^{19.5} \mathrm{eV}$ have a considerable probability of conversion into a pair (more than 5\%) 19]. For

energies below $10^{19.5} \mathrm{eV}$ the probability conversion is small and no difference is seen in the aperture calculation for simulation with and without the pre shower effect.

The probability conversion increases very quickly and nears $100 \%$ between $10^{20.0}$ and $10^{20.5} \mathrm{eV}$ depending on the arrival direction of the particles in relative to the Earth magnetic field. The aperture calculation shown in Fig. 11 illustrates the increase of the conversion probability. Gamma showers simulated with the pre shower effect evolve from a "gamma without preshower profile" to a "hadronic profile" as the energy varies from $10^{19.5}$ to $10^{20.6}$ $\mathrm{eV}$ making the HiRes-I aperture for gamma showers close to the aperture for the hadronic shower.

Nevertheless, even at $10^{20.6} \mathrm{eV}$, where the conversion probability of a gamma in the Earth magnetic field has already reached $100 \%$ the HiRes-I telescope aperture for gamma showers is smaller than the aperture for proton or iron nuclei showers.

The effects of the variation of the aperture with energy for different particle type is taken into account in the next section in order to calculate the energy spectrum. 


\section{SPECTRUM}

The fact that the HiRes aperture has been calculated under the assumption of hadronic primaries, opens the possibility of the existence of systematic effects for a broad range of cosmic ray production scenarios.

In particular, it cannot be disregarded at present the possibility of mixed extragalactic components: a hadronic one, coming from conservative bottom-up acceleration mechanisms and a harder component, dominated by photons, originated in more exotic top-down models.

From the myriad of top-down mechanisms suggested to explain the observed trans-GZK events [33], the two most promising involve the decay or annihilation of topological defects and superheavy dark matter [34, 35]. It has been demonstrated by [36] that the energy spectrum of ultra-high energy cosmic rays generated from superheavy particles and topological defects is approximately proportional to $E^{-1.9}$ and that, even if the $\gamma / N$ ratio is substantially larger than suggested by previous calculations, photons are dominant above $7-8 \times 10^{19} \mathrm{eV}$. Superheavy dark matter and several of the topological defects (e.g., monopolonia and vortons) cluster strongly in galactic halos and, therefore, their spectrum at Earth is dominated by the gamma production spectrum of particles originated in our own halo. Consequently, the energy spectrum can be approximated by a power law of spectral index $\sim 2$.

On the other hand, it has been argued by 36] that necklaces, instead of clustering in the Halo, distribute homogeneously with a rather small separation, of the order of a few tens of kpc. This means that the energy spectrum is modified by interactions with the cosmic microwave background. There is a progressive decrease of the $\gamma / N$ fraction as energy decreases and protons become dominant below few $\times 10^{20} \mathrm{eV}$. The resultant spectrum above $\sim 3 \times 10^{20} \mathrm{eV}$ resembles the GZK structure, but softened and with a rapid recovery.

From the two cases discussed above, clustered and non-clustered top-down primaries, those that do not cluster are the ones that best fit the AGASA energy spectrum.

In any case, top-down models can only account for the highest energy particles, but a more conventional bottom-up model is needed to explain the rest of the extragalactic spectrum.

As an example, for the bottom-up component, we consider proton primaries produced by an isotropic distribution of evolving astrophysical sources.

Indeed, it has been show by [37] that regular astrophysical sources, like active galactic 
nuclei, can naturally explain the observed energy spectrum between 1 and $80 \mathrm{EeV}$.

The energy losses of protons in the intergalactic medium are mainly due to interactions with the comic microwave radiation and, to a smaller degree, from radio and infrared backgrounds [38, 39]. The dominant contributions come from the expansion of the universe, electron-positron pair production and photo-pion production.

We characterize energy losses by using the results from [37], which are essentially indistinguishable from those of [40] calculated with the detailed MC code SOPHIA, and use the formulation of [41]. The source luminosity is assumed to have a general redshift dependence of the form $L=L_{0}(1+z)^{m}$.

As an example, Fig. 12 illustrates a combined bottom-up/top-down spectrum. The GZKed spectrum (lower thick line) has been numerically calculated using a uniform distribution of cosmological sources which extends to a maximum redshift $z_{\max }=5$ and whose luminosity evolves with redshift according to $m=4$. (Actually, since we are only interested on energies above $10^{19} \mathrm{eV}$, the value of of $z_{\max }$ is not important as long as it is larger than 4.) Protons were injected at the sources, with a power law spectrum $d N_{p} / d E \propto E^{-2.5}$ ) extending to arbitrary large energies $\left(E_{\max }=\infty\right)$. The resultant spectrum is normalize to the flux observed by AGASA at $10^{19} \mathrm{eV}$. The top-down spectrum is assumed dominated by photons, $d N_{\gamma} / d E \propto E^{-2}$, as expected from the discussion above. The normalization of the latter spectrum is such that trans-GZK AGASA data can be fitted by the combined spectrum (thick dashed line).

The dot dashed curves illustrate the spectrum that HiRes would infer for the case of a photon component described with and without pre-showering. It can be seen that in the case without pre-shower the effect could be severe enough as to make AGASA and HiRes spectra compatible within quoted uncertainties. The existence of pre-showers considerably diminishes this effect, maintaining unaltered the AGASA-HiRes discrepancy. Although only one realization of the mixed scenario is shown, the conclusions are not altered by changes in the power index of the generation spectrum of protons, the evolution rate $m$, maximum injected energy, $E_{\text {max }}$, or reasonable variations in the hardness of the top-down gamma spectrum. 


\section{CONCLUSION}

The HiRes-I telescope aperture was calculated for different primary particles (iron nuclei, proton and gamma with and without the pre-shower effect) and different simulation codes (CORSIKA and CONEX). The agreement of our calculations with the results published by the HiRes Collaboration for proton primaries is shown in Fig. 9] Same figure shows the agreement between the CONEX and CORSIKA simulation programs.

The recently released program CONEX has shown to be a useful tool for aperture calculations where a great number of events need to be simulated. We have tested the effect of using the same longitudinal profile several time by randomly drawing a new geometry and we concluded that no significant bias is introduced by this procedure.

We calculated the fluctuations in the aperture determination as a function of the number of simulated showers and energy. We have shown that to keep the one sigma fluctuations below $6 \%$ for energies varying from $10^{19}$ to $10^{20.6} \mathrm{eV}$ it is needed to simulate at least 2500 shower.

The aperture for gamma showers was calculated for the HiRes-I telescope and has shown to be a factor 1.5 smaller than the aperture for proton or iron nuclei shower for energies below $10^{19.5} \mathrm{eV}$. For energies above $10^{19.5} \mathrm{eV}$, the pre-shower effect becomes important and changes completely the scenario. Gamma showers develop earlier in the atmosphere due to the interaction with the Earth magnetic field and the efficiency of detection of the telescopes increases. The resultant aperture rises getting closer to the aperture for proton shower. However, even at $10^{20.6} \mathrm{eV}$ the telescope aperture for gammas is a factor 1.2 smaller than the aperture for protons.

The spectrum corresponding to the HiRes-I telescope aperture for gamma and protons supposing a mixed bottom-up and top-down component is shown in Fig 12. The differences in aperture for gamma and proton showers is not enough to explain the differences between the AGASA and HiRes spectra. If the pre-shower effect was not taken into account the AGASA and HiRes spectra would be compatible within the quoted uncertainties. 


\section{ACKNOWLEDGMENTS}

This work was supported by the Brazilian science foundations FAPESP and CNPq to which we are grateful. Most simulations were carried out on a Cluster Linux TDI, supported by Laboratório de Computação Científica Avançada at Universidade de São Paulo.

[1] K. Greisen, Phys. Rev. Lett. 16 (1966) 748.

[2] G. T. Zatsepin, V. A. Kuz'min, ZhETF Pis'ma 4 (1966) 114.

[3] T. Stanev, Phys. Rev. Lett. 77 (1996) 3708.

[4] M. Takeda, et al., Phys. Rev. Lett. 73 (1994) 3491.

[5] T. Abu-Zayyad, et al., Nucl. Instr. Meth. A 450 (2000) 253.

[6] R. U. Abbasi, et al., Phys. Rev. Lett. 92 (2004) 151101.

[7] T. K. Gaisser, A. M. Hillas, 15 ${ }^{\text {th }}$ Int. Cosmic Ray Conf. 8, Plovdiv (Bulgaria) (1977) 353.

[8] T. Abu-Zayyad, et al., Astropart. Phys. 16 (2001) 1.

[9] C. Song, Z. Cao, B. R. Dawson, B. E. Fick, P. Sokolsky, X. Zhang, Astropart. Phys. 14 (2000) 7.

[10] C. L. Pryke, Astropart. Phys. 14 (2001) 319.

[11] R. U. Abbasi, et al., Astropart. Phys. 23 (2005) 157.

[12] T. Abu-Zayyad, et al., Astrophys. J. 557 (2001) 686.

[13] M. Risse, et al., Astropart. Phys. 21 (2004) 479.

[14] P. Homola, et al., Acta Phys. Polon. B 35 (2004) 1893.

[15] P. Homola, et al., astro-ph/0411060 (2004).

[16] M. Risse, et al., astro-ph/0502418 (2005).

[17] D. Heck, J. Knapp, J. Capdevielle, G. Schatz, T. Thouw., Forschungszentrum Karlsruhe, Report FZKA 6019 (1998).

[18] N. N. Kalmykov, S. S. Ostapchenko, A. I. Pavlov, Nucl. Phys. B (Proc. Suppl.) 52B (1997) 17.

[19] P. Homola, et al., astro-ph/0311442 (2003).

[20] National Geophysical Space Center; http://www.ngdc.noaa.gov/seg/geomag/.

[21] T. Pierog, et al., astro-ph/0411260 (2004). 
[22] G. Bossard, et al., Phys. Rev. D 63 (2001) 054030.

[23] J. A. Ortiz, G. Medina-Tanco, V. de Souza, Astropart. Phys. 23 (2005) 463.

[24] F. Kakimoto, et al., Nucl. Instr. Meth. A 372 (1996) 527.

[25] M. Risse, D. Heck, Astropart. Phys. 20 (2004) 661.

[26] M. Nagano, K. Kobayakawa, N. Sakaki, K. Ando, Astropart. Phys. 22 (2004) 235.

[27] B. Dawson, Auger Technical Note, GAP-02-067 (2002).

[28] R. M. Baltrusaitis, et al., Nucl. Instr. Meth. A 240 (1985) 410.

[29] A. N. Bunner, Cosmic ray detection by atmospheric fluorescence, Ph.D. Thesis, Cornel University (1967).

[30] C. Song, Study of ultra high energy cosmic rays with the high resolution fly's eye prototype detector, Ph.D. Thesis, Columbia University (2001).

[31] J. Bellido, Anisotropy studies of the HiRes EHECR, Ph.D. Thesis, University of Adelaide (2002).

[32] H. J. Drescher, G. R. Farrar, Phys. Rev. D 67 (2003) 116001.

[33] P. Bhattacharjee, G. Sigl, Physics Reports 327 (2000) 109.

[34] V. Berezinsky, Nucl. Phys. (Proc. Suppl) B87 (2000) 387.

[35] V. Kuzmin, I. Tkachev, Phys. Rep. 320 (1999) 199.

[36] R. Aloisio, V. Berezinsky, M. Kachelries, Nuclear Physics B (Proc. Suppl.) 136 (2004) 319, astro-ph/0409222.

[37] V. Berezinsky, A. Gazizov, S. Grigorieva, astro-ph/0204357.

[38] V. Berezinskii, S. V. Bulanov, V. A. Dogiel, V. L. Ginzburg, V. Ptuskin, Astrophysics of Cosmic Rays, North-Holland, 1990.

[39] J. P. Rachen, Interaction processes and statistical properties of the propagation of cosmic rays in photon backgrounds, PhD Thesis, Rheinschen Friedrich-Wilhelms-Universitat, Bonn, 1996.

[40] T.Stanev, R.Engel, A.Muecke, R.J.Protheroe, J.P.Rachen, Phys. Rev. D62 (2000) 093005.

[41] V.S.Berezinsky, S.I.Grigorieva, Astron. \& Astroph. 199 (1988) 1. 


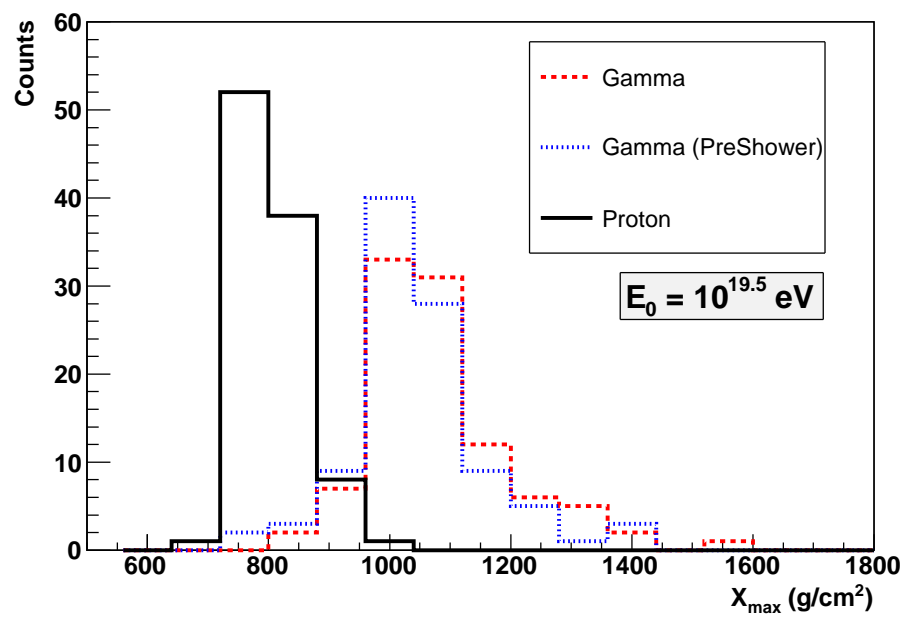

FIG. 1: Distribution of $X_{\max }$ for showers initiated by proton and gamma at $10^{19.5} \mathrm{eV}$. Gamma shower were simulated with and without the pre-shower effect. For each energy and primary we simulated 100 showers.

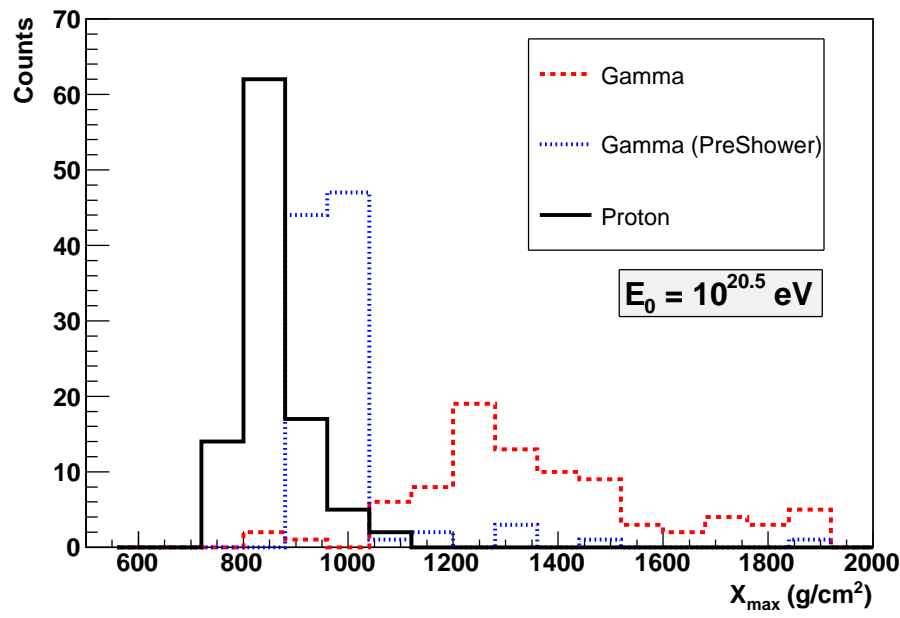

FIG. 2: Distribution of $X_{\max }$ for showers initiated by proton and gamma at $10^{20.5} \mathrm{eV}$. Gamma shower were simulated with and without the pre-shower effect. For each energy and primary we simulated 100 showers. 


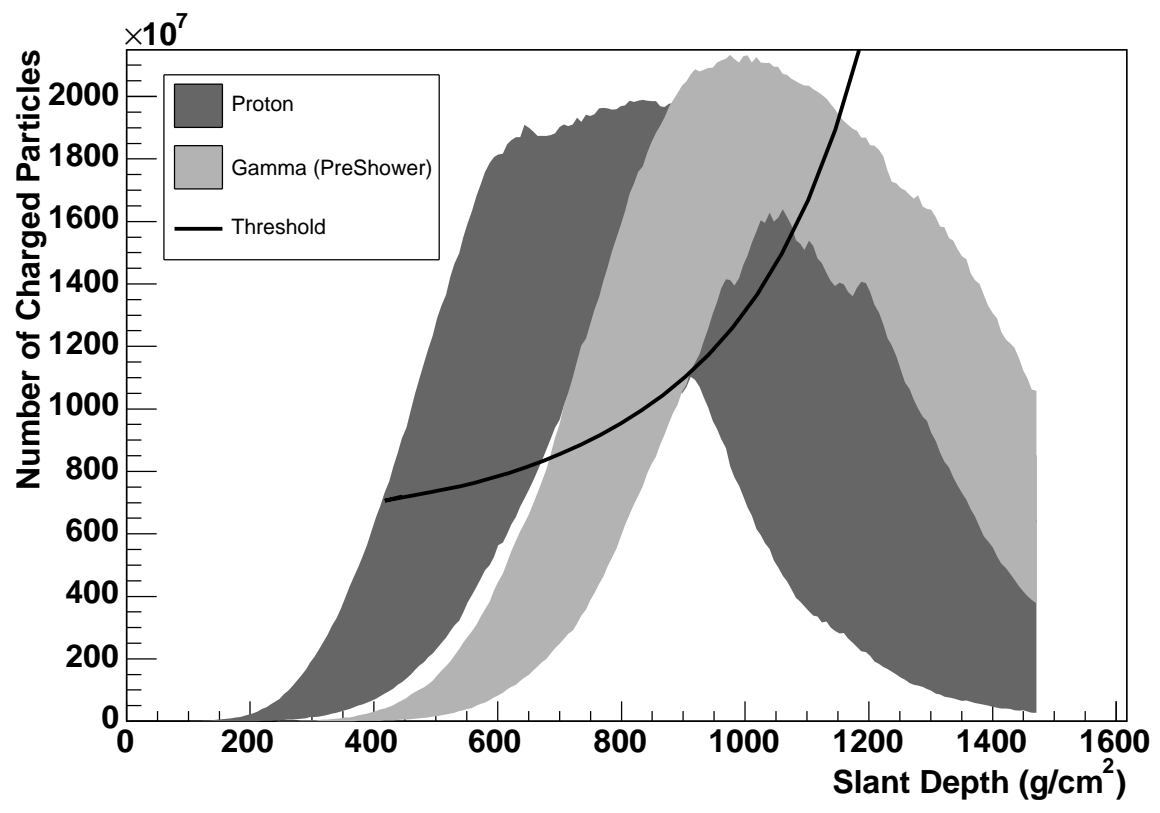

FIG. 3: Distribution of $X_{\max }$ for showers initiated by proton and gamma at $10^{19.5}$ and $10^{20.5}$ eV. Gamma shower were simulated with and without the pre-shower effect. For each energy and primary we simulated 100 showers.

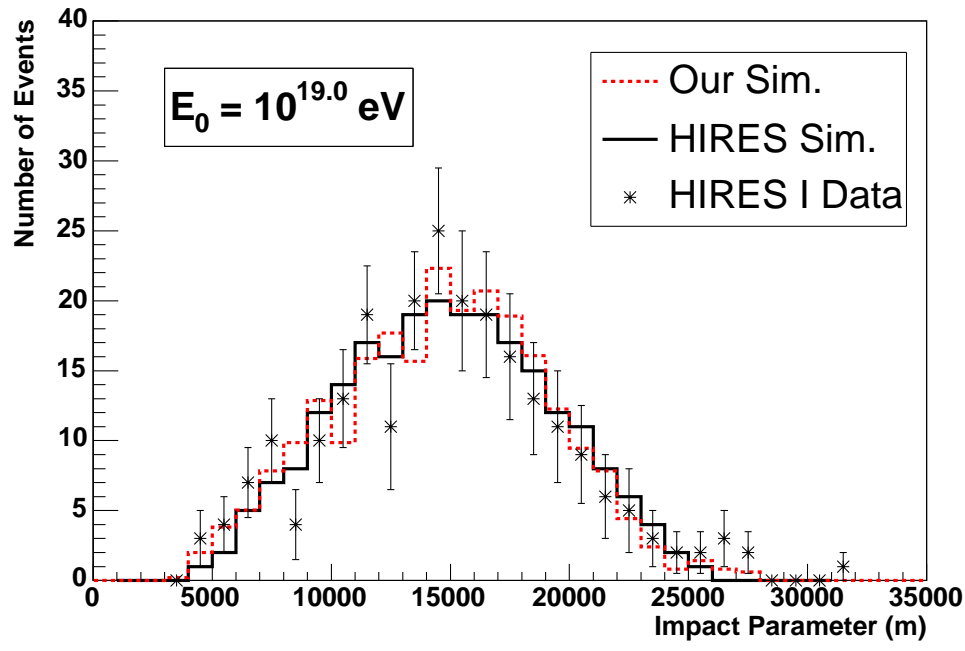

FIG. 4: Distribution of impact parameter for shower initiated with energies $10^{19} \mathrm{eV}$. We compare our simulation with the HiRes-I data and the Hires Collaboration Simulation. The $\chi^{2}$ between the HiRes data and the HiRes simulation is 1.6 and the $\chi^{2}$ between the HiRes data and our simulation is 1.7. The HiRes data and simulation have been extracted from [6]. 


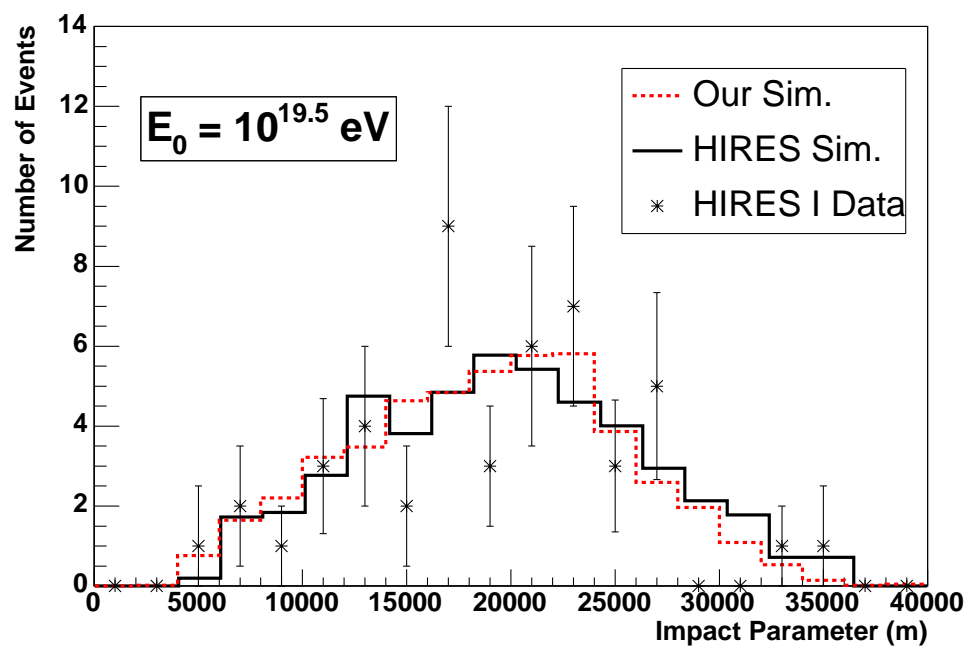

FIG. 5: Distribution of impact parameter for shower initiated with energies $10^{19.5} \mathrm{eV}$. We compare our simulation with the HiRes-I data and the Hires Collaboration Simulation. The $\chi^{2}$ between the HiRes data and the HiRes simulation is 1.5 and the $\chi^{2}$ between the HiRes data and our simulation is also 1.5. The HiRes data and simulation have been extracted from $[\underline{6}]$.

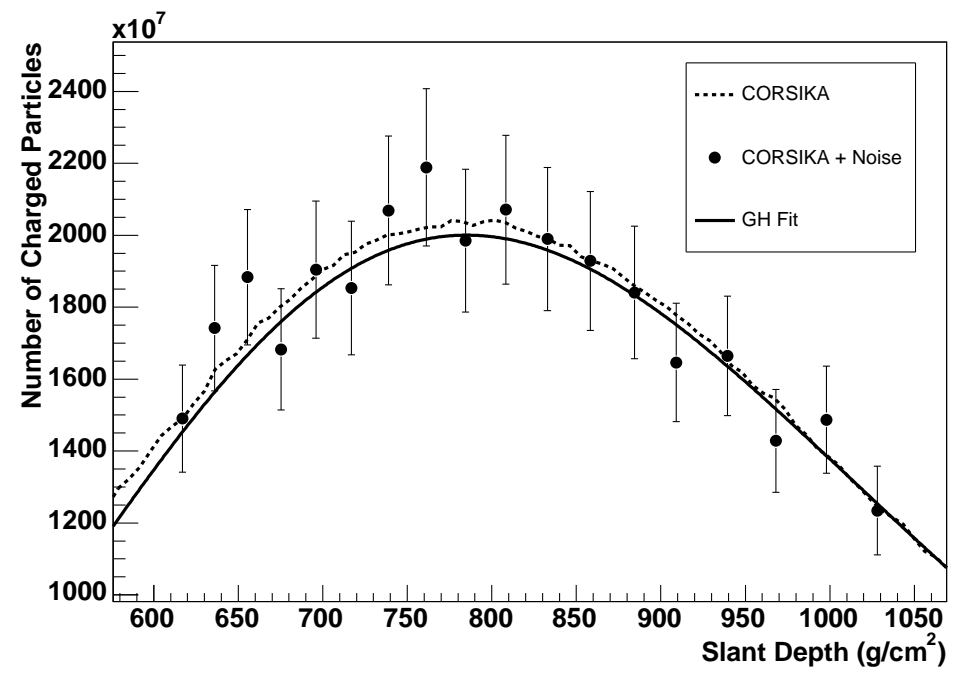

FIG. 6: Longitudinal particles profile simulated by CORSIKA, after detector simulation and reconstructed Gaisser-Hillas profile. 


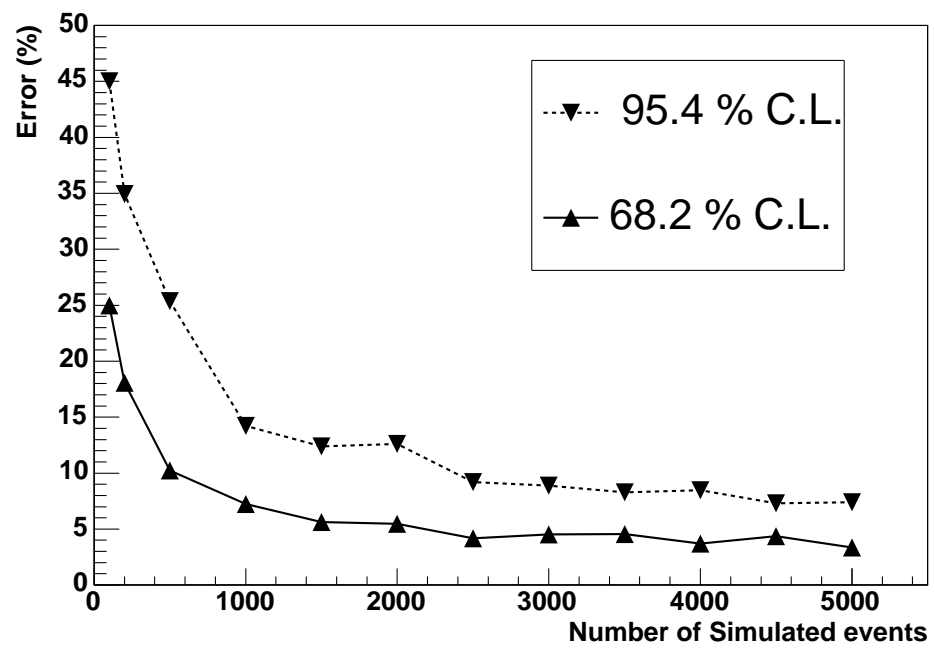

FIG. 7: Error of the aperture as a function of the total number of events simulated.

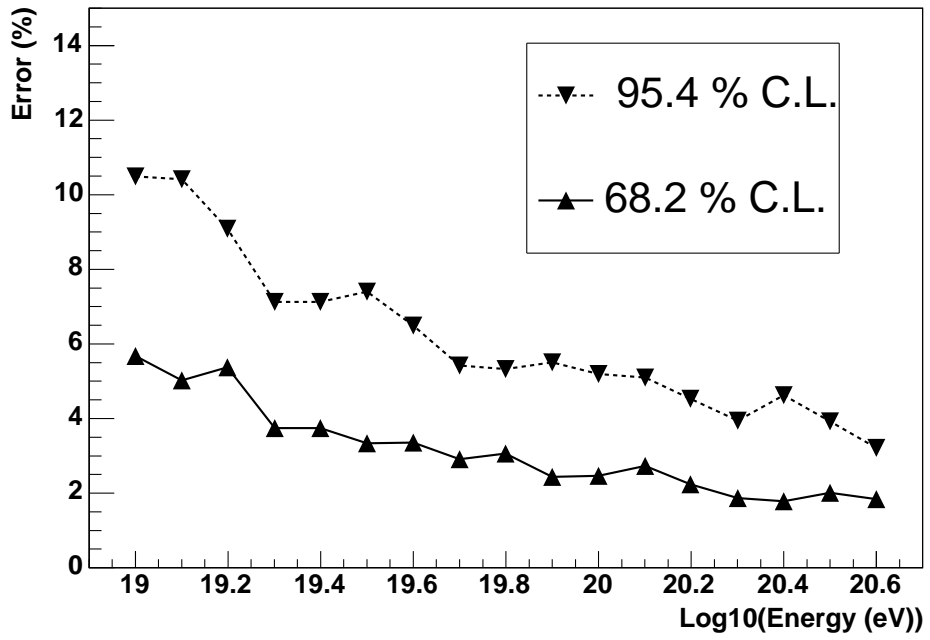

FIG. 8: Error of the aperture as a function of the shower energy. We have used 5000 proton shower for each energy. 


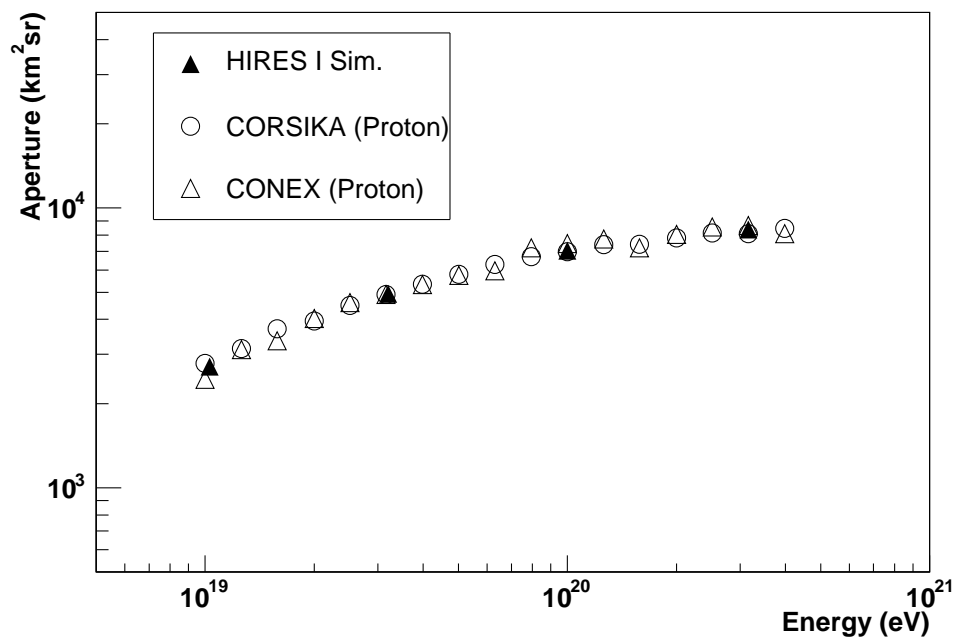

FIG. 9: HiRes-I telescope aperture calculated by the HiRes-I Collaboration and published in reference [6], calculated with our analysis program using shower generated by the CORSIKA and CONEX codes.

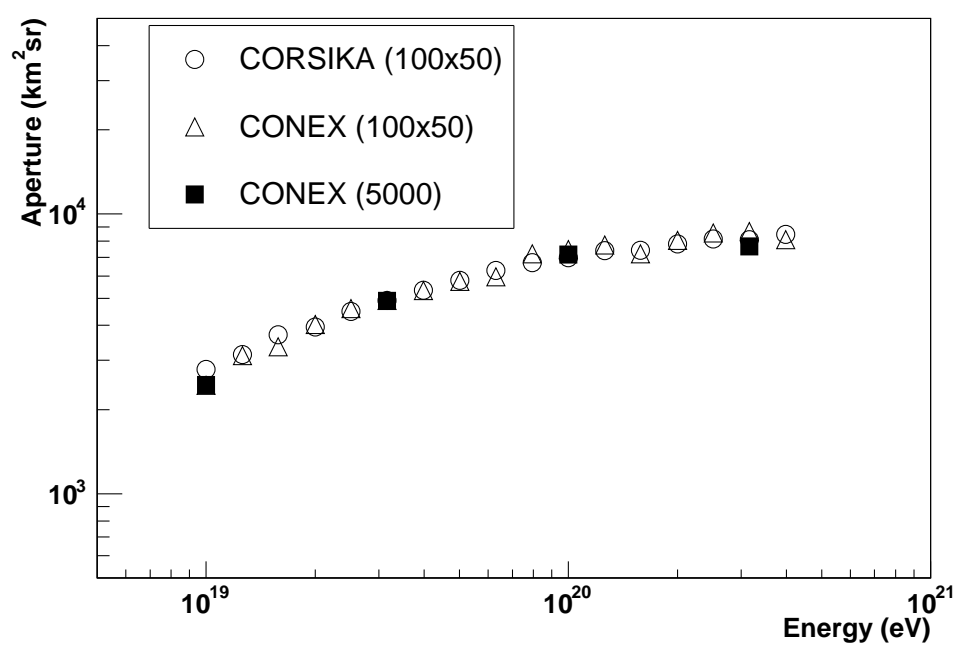

FIG. 10: HiRes-I telescope aperture calculated calculated with our analysis program using showers generated by the CORSIKA and CONEX codes in two approaches: a) (100x50) simulate 100 different shower and use each one 50 times by ramdonly choosing a different geometry and b) (5000) simulate 5000 different showers 


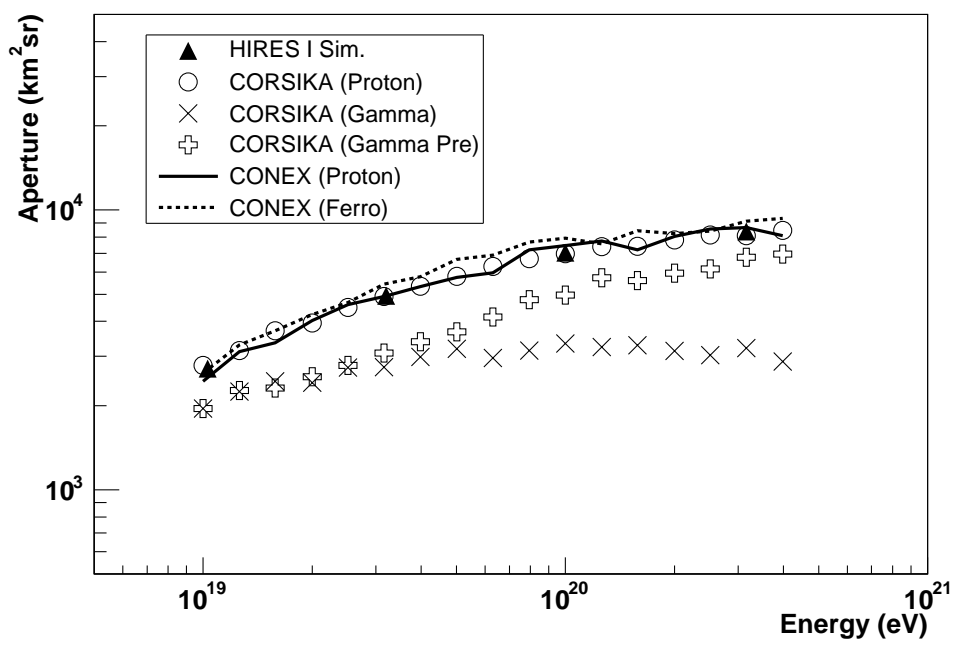

FIG. 11: HiRes-I telescope aperture calculated by the HiRes-I Collaboration and published in reference [6], calculated with our analysis program using showers generated by the CORSIKA and CONEX codes. 


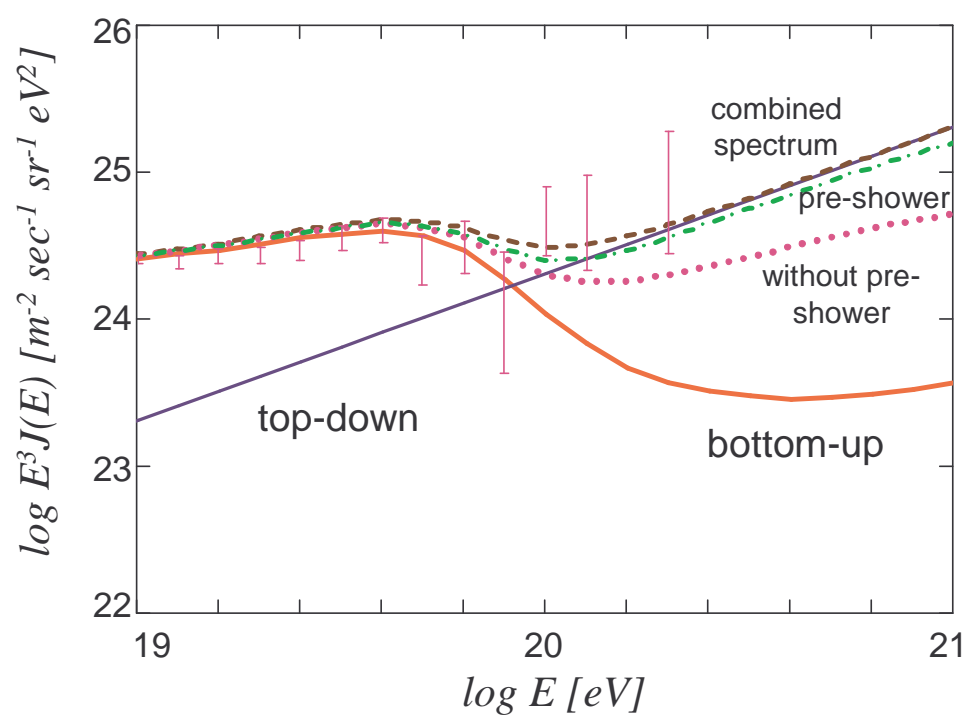

FIG. 12: Cosmic ray spectrum as seen by the HiRes-I telescope for different primary particles and production scenarios (see details in the text), depending whether pre-showering is included or not in the calculation of the aperture. 